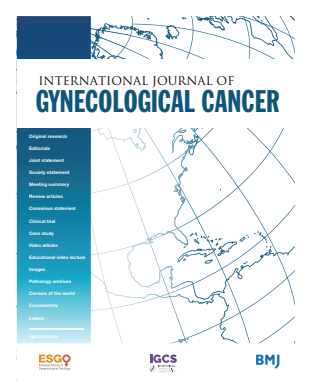

\title{
ConCerv: a prospective trial of conservative surgery for low-risk early-stage cervical cancer
}

Kathleen M Schmeler (D) , ${ }^{1}$ Rene Pareja (D) , ${ }^{2}$ Aldo Lopez Blanco, ${ }^{3}$ Jose Humberto Fregnani, ${ }^{4}$ Andre Lopes, ${ }^{5}$ Myriam Perrotta, ${ }^{6}$ Audrey T Tsunoda, ${ }^{7}$ David F Cantú-de-León, ${ }^{8}$ Lois M Ramondetta, ${ }^{1}$ Tarinee Manchana, ${ }^{9}$ David R Crotzer, ${ }^{10}$ Orla M McNally, ${ }^{11}$ Martin Riege,${ }^{12}$ Giovanni Scambia,${ }^{13}$ Juan Manuel Carvajal, ${ }^{14}$ Julian Di Guilmi, ${ }^{15}$ Gabriel J Rendon (D) ${ }^{16}$ Preetha Ramalingam, ${ }^{17}$ Bryan M Fellman, ${ }^{18}$ Robert L Coleman, ${ }^{19}$ Michael Frumovitz (i) , ${ }^{1}$ Pedro T Ramirez ${ }^{1}$

For numbered affiliations see end of article.

\section{Correspondence to} Dr Kathleen M Schmeler, Gynecologic Oncology, The University of Texas MD Anderson Cancer Center, Houston, TX 77230, USA; KSchmele@mdanderson.org

For 'Presented at statement' see end of article.

Received 3 July 2021 Accepted 11 August 2021 Published Online First 7 September 2021

\section{Linked}

- http://dx.doi.org/10.1136/ ijgc-2021-003008

Check for updates

(C) IGCS and ESGO 2021. No commercial re-use. See rights and permissions. Published by BMJ.

\footnotetext{
To cite: Schmeler KM, Pareja R, Lopez Blanco A, et al. Int J Gynecol Cancer 2021;31:1317-1325.
}

\section{HIGHLIGHTS}

- Conservative surgery was associated with a 3.5\% recurrence rate in women with low-risk cervical cancer.

- The rate of positive lymph nodes was $5 \%$, with lymph node assessment recommended in this low-risk population.

- Further study is needed to determine long-term outcomes and optimal pathologic criteria for conservative surgery.

\section{ABSTRACT}

Objective The objective of the ConCerv Trial was to prospectively evaluate the feasibility of conservative surgery in women with early-stage, low-risk cervical cancer.

Methods From April 2010 to March 2019, a prospective, single-arm, multicenter study evaluated conservative surgery in participants from 16 sites in nine countries. Eligibility criteria included: (1) FIGO 2009 stage IA2-IB1 cervical carcinoma; (2) squamous cell (any grade) or adenocarcinoma (grade 1 or 2 only) histology; (3) tumor size $\leq 2 \mathrm{~cm}$; (4) no lymphovascular space invasion; (5) depth of invasion $\leq 10 \mathrm{~mm}$; (6) negative imaging for metastatic disease; and (7) negative conization margins. Cervical conization was performed to determine eligibility, with one repeat cone permitted. Eligible women desiring fertility preservation underwent a second surgery with pelvic lymph node assessment, consisting of sentinel lymph node biopsy and/or full pelvic lymph node dissection. Those not desiring fertility preservation underwent simple hysterectomy with lymph node assessment. Women who had undergone an 'inadvertent' simple hysterectomy with an unexpected post-operative diagnosis of cancer were also eligible if they met the above inclusion criteria and underwent a second surgery with pelvic lymph node dissection only.

Results 100 evaluable patients were enrolled. Median age at surgery was 38 years (range 23-67). Stage was IA2 (33\%) and IB1 (67\%). Surgery included conization followed by lymph node assessment in 44 women, conization followed by simple hysterectomy with lymph node assessment in 40 women, and inadvertent simple hysterectomy followed by lymph node dissection in 16 women. Positive lymph nodes were noted in 5 patients (5\%). Residual disease in the post-conization hysterectomy specimen was noted in $1 / 40$ patients-that is, an immediate failure rate of $2.5 \%$. Median follow-up was 36.3 months (range 0.0-68.3). Three patients developed recurrent disease within 2 years of surgery - that is, a cumulative incidence of $3.5 \%$ (95\% $\mathrm{Cl} 0.9 \%$ to $9.0 \%)$.
Discussion Our prospective data show that select patients with early-stage, low-risk cervical carcinoma may be offered conservative surgery.

\section{INTRODUCTION}

Approximately 570000 new cases of cervical cancer and 311000 related deaths occur annually worldwide. ${ }^{1}$ About $85 \%$ of these cases and deaths occur in low- and middle-income countries. ${ }^{2}$ Cervical cancer screening programs have led to a significant reduction in the incidence and mortality of cervical cancer in high-income countries. By contrast, the cervical cancer burden remains unchanged in low- and middle-income countries, primarily due to a lack of effective organized programs for cervical screening and treatment of pre-invasive disease. The World Health Organization (WHO) recently implemented a global strategy for the elimination of cervical cancer as a public health problem. The 2030 goals of the program include: (1) $90 \%$ of girls to receive complete human papillomavirus vaccination by age 15 , (2) $70 \%$ of women to undergo cervical cancer screening with a high performance test at 35 and 45 years of age, and (3) $90 \%$ of women with pre-invasive or invasive cervical lesions to undergo treatment. ${ }^{3}$ If successfully implemented, these aggressive efforts will result in the majority of women around the world being diagnosed with pre-invasive or early-stage cervical cancer that can be treated and cured.

For women with early-stage cervical cancer, the current standard treatment is a radical hysterectomy with removal of the uterus, cervix, upper vagina, and parametrium as well as the pelvic lymph nodes. ${ }^{4}$ In women who desire fertility preservation, a radical trachelectomy is an acceptable alternative, with equivalent oncologic outcomes. ${ }^{5}$ This consists of 


\section{Original research}

removal of the cervix, upper vagina, and parametrium while sparing the uterine fundus, allowing for future pregnancy. Although radical hysterectomy and radical trachelectomy result in excellent local tumor control, they can be associated with significant morbidity due to removal of the parametrium, which contains autonomic nerve fibers associated with bladder, bowel, and sexual function. ${ }^{6-10}$ These radical procedures are also associated with surgical complications, such as hemorrhage, bladder and ureteral injury, and fistula formation. Furthermore, these procedures require a provider with specialized training in gynecologic oncology surgery, often not available in many low- and middle-income countries.

In recent years, the usefulness of parametrial resection in women with early-stage cervical cancer has come under question. Several retrospective studies have reported that $<1 \%$ of women with earlystage disease and favorable pathologic characteristics (tumor $\leq 2 \mathrm{~cm}$, depth of invasion $\leq 10 \mathrm{~mm}$, and negative pelvic nodes) have parametrial involvement. ${ }^{11-15}$ In addition, several retrospective and small prospective studies have shown favorable results with conservative surgery consisting of cervical conization or simple hysterectomy, with lymph node assessment in select women with low-risk cervical cancer. ${ }^{16-22}$ To further evaluate the oncologic outcomes of conservative surgery, we performed the ConCerv Trial, the first prospective study of conservative surgery in women with early-stage, low-risk cervical cancer.

\section{METHODS}

The ConCerv Trial was a prospective, single-arm, multicenter study to evaluate the feasibility and oncologic outcomes of conization alone or simple hysterectomy in women with early-stage, low-risk cervical carcinoma. Institutional review board approval was obtained from the University of Texas MD Anderson Cancer Center (protocol 2008-0118, NCT01048853) and all participating institutions. Eligibility criteria included: (1) FIG0 2009 stage IA2-IB1 cervical carcinoma; (2) squamous cell (any grade) or adenocarcinoma (grade 1 or 2 only) histology; (3) tumor size $\leq 2 \mathrm{~cm}$ by physical examination and/or imaging studies; (4) no lymphovascular space invasion; (5) negative imaging for metastatic disease with CT scan, MRI, and/or positron emission tomography scan; (6) depth of invasion $\leq 10 \mathrm{~mm}$; and (7) conization margins and endocervical curettage negative for malignancy and high-grade dysplasia. A negative margin was defined as no invasive cancer within $1.0 \mathrm{~mm}$ of both the endocervical and ectocervical margins and no adenocarcinoma in situ, cervical intraepithelial neoplasia 2 or 3 at the inked or cauterized margin. Inclusion criteria 6 and 7 were added after the first year of the study as described in the Results section.

All patients provided informed consent for the study and underwent a cervical conization and endocervical curettage to determine eligibility. Of note, women who had undergone conization at an outside institution were considered eligible if they met the inclusion criteria. In all cases, one repeat conization and endocervical curettage was permitted if required to meet the inclusion criteria. Eligible women desiring fertility preservation underwent a second surgery with pelvic lymph node assessment, consisting of sentinel lymph node biopsy and/or full pelvic lymph node dissection based on each participating institution's guidelines and standard practices. Those not desiring fertility preservation underwent a second surgery with simple hysterectomy and pelvic lymph node assessment. Patients who had undergone an inadvertent simple hysterectomy with an unexpected post-operative diagnosis of invasive cancer were also eligible if they met the above inclusion criteria and had negative margins on the hysterectomy specimen. These patients underwent a second surgery with pelvic lymph node dissection only. All pathologic specimens were centrally reviewed by an expert gynecologic pathologist at MD Anderson Cancer Center (PR). This included review of cone and inadvertent hysterectomy specimens to confirm eligibility prior to undergoing simple hysterectomy and/or lymph node assessment. In addition, all final hysterectomy and lymph node specimens were centrally reviewed. Frozen section for the conization specimens was not permitted due to the requirement for final pathologic analysis, including central pathology review, prior to performing definitive conservative surgery.

Surgery could be performed using an open, laparoscopic, or robotic approach based on each participating institution's standard practice and surgeon preference. Post-operatively, study participants were followed with pelvic examination and cytology every 3 months for 2 years, and then according to local standard of care. Quality of life factors, sexual functioning, and satisfaction with healthcare decisions were assessed prior to surgery at $3,6,12$ and 24 months following surgery, and will be reported in a separate publication.

The primary objective of the study was to evaluate the feasibility of performing, and oncologic outcomes of, conservative surgery. We determined the immediate failure rate, defined as residual disease in the simple hysterectomy specimen of women who underwent conization followed by simple hysterectomy and lymph node assessment. Futility monitoring of feasibility was performed throughout the study using the Bayesian methods of Thall et al. ${ }^{23}$ The proposed treatment strategy was considered infeasible if there was more than an $80 \%$ chance that the immediate failure rate exceeded $3 \%$. If this was reached, the trial would be stopped. We also evaluated the cervical cancer recurrence rate at 2 years, with an additional stopping rule stating that the study would be discontinued if two or more patients developed recurrent disease within this time period. We assumed a beta $(0.15,4.85)$ prior distribution for the immediate failure rate. The trial was designed with a sample size of 100 subjects to have desirable operating characteristics. We also evaluated the rate of pelvic lymph node positivity and quality of life outcomes. The overall conduct of the study was monitored by the MD Anderson Cancer Center Data and Safety Monitoring Committee.

Frequencies were used to describe the number of enrolled, eligible and evaluable patients by institutional site. Standard summary statistics were used to describe the clinical and demographic characteristics of the evaluable study population. We estimated the immediate failure rate (residual disease) along with a $90 \%$ credible interval. We also reported the posterior probability that the immediate failure rate is $3 \%$ or more. We estimated the 2 -year cumulative incidence of recurrence in the study population along with $95 \%$ confidence intervals. Cumulative incidence of recurrence was measured from the date of surgery to the earliest date of the last clinic visit, date of first recurrence, or date of death. Death was considered a competing event for recurrence. Recurrence-free survival was estimated using the methods of Kaplan and Meier, and was measured from the date of surgery to the earliest date 
Table 1 Study accrual by participating site

\begin{tabular}{llll}
\hline Institution & City & Country & Number of evaluable participants \\
\hline MD Anderson Cancer Center & Houston & USA & $36(36 \%)$ \\
Instituto de Cancerología & Medellin & Colombia & $14(14 \%)$ \\
Instituto Nacional de Enfermedades Neoplásicas & Lima & Perú & $13(13 \%)$ \\
Barretos Cancer Hospital & Barretos & Brazil & $8(8 \%)$ \\
Hospital Italiano & Buenos Aires & Argentina & $6(6 \%)$ \\
Instituto Brasileiro de Controle do Cancer & São Paulo & Brazil & $6(6 \%)$ \\
Hospital Erasto Gaertner & Curitiba & Brazil & $5(5 \%)$ \\
Instituto Nacional de Cancerologia & Mexico City & México & $4(4 \%)$ \\
Lyndon B. Johnson Hospital/Harris Health & Houston & USA & $3(3 \%)$ \\
Chulalongkorn University & Bangkok & Thailand & $1(1 \%)$ \\
Royal Women's Hospital & Melbourne & Australia & $1(1 \%)$ \\
Nebraska Methodist Health System & Omaha & USA & $1(1 \%)$ \\
Instituto de Ginecología de Rosario & Rosario & Argentina & $1(1 \%)$ \\
Fondazione Policlinico Universitario A. Gemelli IRCCS & Rome & Italy & $1(1 \%)$ \\
\hline
\end{tabular}

of the last clinic visit, date of first recurrence, or date of death. All statistical analyses were performed using Stata/MP version 16.0 (College Station, Texas USA).

MD Anderson Cancer Center served as the lead site and coordinating center for the ConCerv Trial, providing oversight for all participating sites. All study data were collected and managed using the Research Electronic Data Capture (REDCap) tools hosted at MD Anderson. ${ }^{24}$ REDCap is a secure, web-based application designed to support data capture for research studies. Any adverse events were reported to the MD Anderson coordinating center and classified according to the National Cancer Institute Common Terminology Criteria for Adverse Events version 4.0 (CTCAE version 4.0) for Toxicity and Adverse Event reporting. In accordance with the journal's guidelines, we will provide our data for the reproducibility of this study in other centers if such is requested.

\section{RESULTS}

A total of 100 evaluable women were enrolled between April 2010 and January 2019 from 14 institutions in nine countries (Table 1). Of note, 140 patients were enrolled to reach 100 evaluable patients: 31 were ineligible after central pathology review; seven withdrew from the study prior to surgery; and two had a positive pregnancy test at the time of surgery. The discrepancies in pathology review included presence of lymphovascular space invasion $(n=15,48.4 \%)$; stage IA1 or pre-invasive disease $(n=13,41.9 \%)$; and adenosquamous or adenoid basal histology $(n=3,9.7 \%)$. Participant demographic and pathologic information are shown in Table 2. The median age at surgery was 38 years (range 23-67). Stage at diagnosis was IA2 (33\%) and IB1 (67\%). Histologic type included squamous cell carcinoma (48\%) and adenocarcinoma (52\%).

The study results are shown in Figure 1. A total of 44 participants $(44 \%)$ desired fertility preservation and underwent cervical conization followed by lymph node assessment. Forty participants (40\%) did not desire fertility preservation and underwent cervical conization followed by simple hysterectomy with lymph node assessment. The remaining 16 participants (16\%) had an inadvertent simple hysterectomy with an unexpected post-operative diagnosis of cancer, followed by lymph node dissection only. Minimally invasive surgery was performed in 96 patients: laparoscopic surgery in 83 patients, and robotic surgery in 13 patients. A full pelvic lymph node dissection was performed in 58 patients $(58 \%)$, sentinel lymph node biopsy and full pelvic lymph node dissection in 38 patients $(38 \%)$, and sentinel biopsy alone in four patients $(4 \%)$. Positive lymph nodes were found in 5 patients $(5 \%)$ (Table 3) who were treated with chemoradiation.

One of 40 patients had residual disease in the hysterectomy specimen after a conization with negative margins, corresponding to an immediate failure rate of $2.5 \%$ (90\% credible interval $0.2-7.2 \%$ ).

Table 2 Patient demographic and pathology information

\begin{tabular}{ll}
\hline Age at surgery (years): & 39 \\
Mean & 38 \\
Median & $23-67$ \\
Range & \\
Stage (FIGO 2009), N (\%) & $33(33 \%)$ \\
IA2 & $67(67 \%)$ \\
IB1 & \\
Histology, N (\%) & $48(48 \%)$ \\
Squamous cell carcinoma & $52(52 \%)$ \\
Adenocarcinoma & \\
Surgical approach, N (\%) & $83(83 \%)$ \\
Laparoscopic & $13(13 \%)$ \\
Robotic & $4(4 \%)$ \\
Open & \\
Lymph node assessment, N (\%) & $58(58 \%)$ \\
Full lymph node dissection & $38(38 \%)$ \\
Sentinel lymph node biopsy+full lymph \\
node dissection \\
Sentinel lymph node biopsy alone \\
\hline
\end{tabular}




\section{Original research}

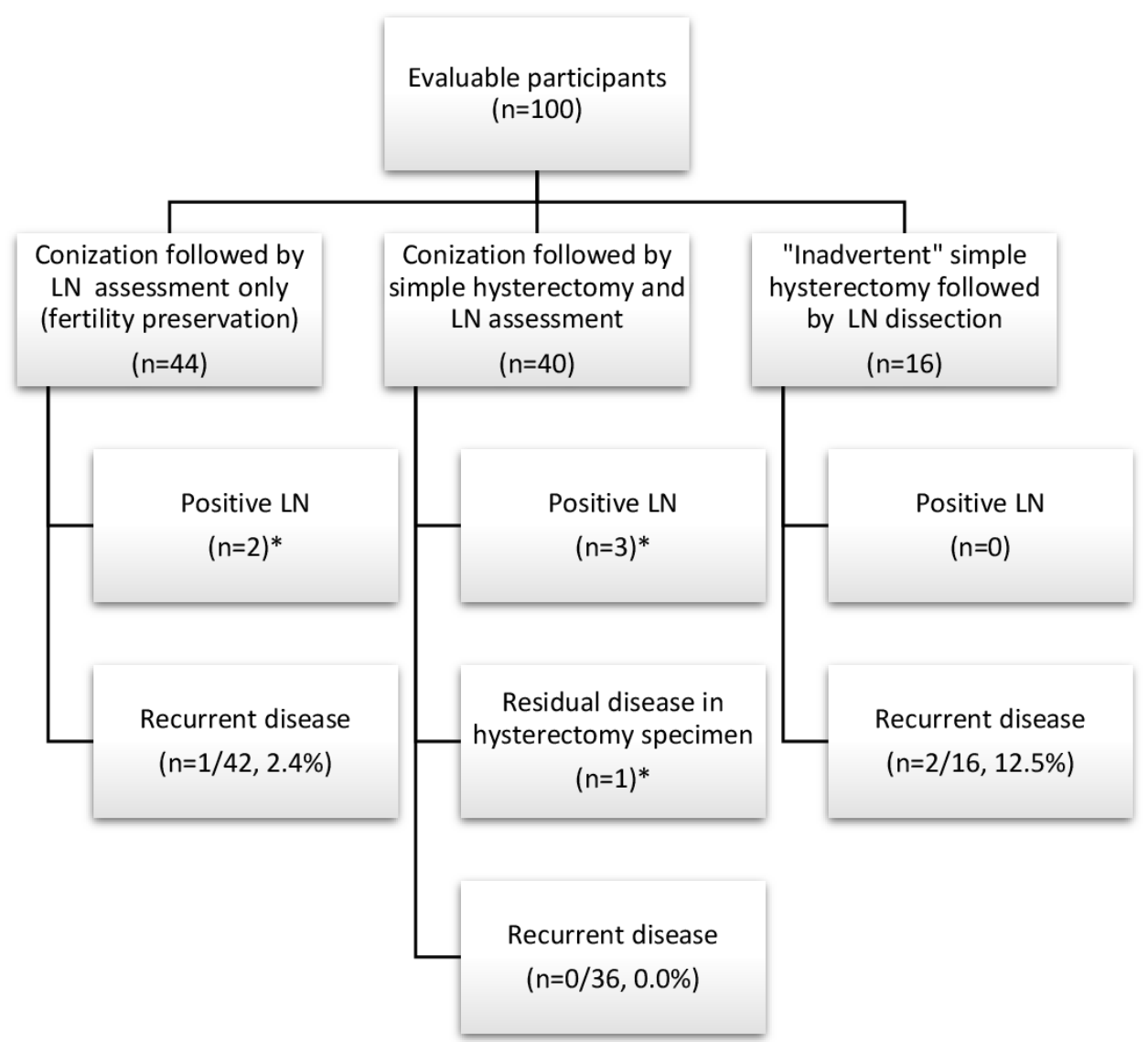

Figure 1 Study results by treatment type. Lymph node (LN), pelvic lymph node assessment with sentinel lymph node biopsy and/or full pelvic lymphadenectomy. *Patients with positive lymph nodes or residual disease in the hysterectomy specimen were excluded from further analyses for rates of recurrent disease.

The posterior probability that the immediate failure rate was greater than $3 \%$ is 0.33 indicating that conservative surgery in this population is feasible with regards to immediate failure. Of note, this patient had a long history of adenocarcinoma in situ followed by a cervical conization which showed a grade 2 adenocarcinoma with $3.0 \mathrm{~mm}$ of invasion with a positive margin. According to protocol, she underwent a repeat conization and endocervical curettage to determine eligibility and both were negative for adenocarcinoma and adenocarcinoma in situ. She subsequently underwent a simple hysterectomy and pelvic lymph node dissection, which showed a $2.0 \mathrm{~mm}$ focus of residual adenocarcinoma in the cervix, with negative margins and negative lymph nodes. She underwent observation and was without evidence of disease 5 years following surgery.

The median follow-up for all participants was 36.3 months (range 0.0-68.3). Three patients developed recurrent disease for a 2-year cumulative incidence of $3.5 \%(95 \% \mathrm{Cl} 0.9 \%$ to $9.0 \%)$. Median recurrence-free survival was not reached. The 2-year recurrence-free survival probability was $0.95(95 \% \mathrm{Cl} 0.88$ to

Table 3 Participants with positive lymph nodes (5/100, 5\%)

\begin{tabular}{|c|c|c|c|c|c|c|}
\hline Patient & Histology & Stage & Visible lesion & Procedure & Depth of invasion & $\begin{array}{l}\text { Number of positive } \\
\text { lymph nodes }\end{array}$ \\
\hline 1 & $\begin{array}{l}\text { Grade } 2 \\
\text { squamous }\end{array}$ & IA2 & No & $\begin{array}{l}\text { Cone } x 2 \\
\text { LND only }\end{array}$ & $\begin{array}{l}4 \mathrm{~mm} \\
0 \mathrm{~mm}\end{array}$ & $1 / 17$ \\
\hline 2 & $\begin{array}{l}\text { Grade } 2 \\
\text { squamous }\end{array}$ & IB1 & No & $\begin{array}{l}\text { Cone } x 2 \\
\text { LND only }\end{array}$ & $\begin{array}{l}6.5 \mathrm{~mm} \\
3.1 \mathrm{~mm}\end{array}$ & $1 / 7$ \\
\hline 3 & Grade 2 squamous & IA2 & No & $\begin{array}{l}\text { Cone } \times 1 \\
\text { SH +LND }\end{array}$ & $3.0 \mathrm{~mm}$ & $1 / 21$ \\
\hline 5 & Grade 2 squamous & IB1 & $\begin{array}{l}\text { Yes } \\
1.8 \mathrm{~cm}\end{array}$ & $\begin{array}{l}\text { Cone x } 1 \\
\text { SH +LND }\end{array}$ & $3.5 \mathrm{~mm}$ & $2 / 28$ \\
\hline
\end{tabular}

All participants with positive lymph nodes were treated with chemoradiation.

LND, lymph node dissection; SH, simple hysterectomy. 
0.98). As shown in Figure 1, the recurrence rate was 1/42 (2.4\%) for evaluable women who underwent cone biopsy alone followed by lymph node assessment; $0 / 36(0.0 \%)$ for women who underwent conization followed by simple hysterectomy and lymph node assessment; and 2/16 (12.5\%) for women who underwent inadvertent simple hysterectomy followed by lymph node dissection. The first recurrence occurred in a patient who desired fertility preservation. She underwent cervical conization and was found to have a grade 2 squamous cell carcinoma with $13 \mathrm{~mm}$ of invasion and positive margins. She underwent a second conization, which was negative for invasive cancer but showed cervical intraepithelial neoplasia 3, which was present at the cone margin. She underwent a laparoscopic pelvic lymphadenectomy with 15 negative lymph nodes. At her 3-month follow-up visit, her cervix appeared normal but cytology showed a high-grade squamous intraepithelial lesion. A cold knife cone biopsy was performed revealing recurrent invasive squamous cell cancer with positive margins. She therefore underwent a radical trachelectomy, which was converted to a radical hysterectomy due to a positive endocervical margin on frozen section. She received adjuvant chemoradiation for highrisk features. She was without evidence of disease at her 5-year follow-up visit. This occurred in the first year of the study and was reviewed by the Data and Safety Monitoring Committee. The inclusion criteria were amended to become more conservative and include a depth of invasion $\leq 10 \mathrm{~mm}$ and negative cone margins for high-grade dysplasia including cervical intraepithelial neoplasia 2/3 and adenocarcinoma in situ.

The other two recurrences were in women who underwent an inadvertent simple hysterectomy. One patient had adenocarcinoma in situ and underwent a cone with negative margins followed by laparoscopic simple hysterectomy which showed an unexpected grade 2 adenocarcinoma with $4.2 \mathrm{~mm}$ of invasion and negative margins. She was enrolled in the trial and underwent laparoscopic lymph node dissection with three negative lymph nodes. Her cancer recurred 11 months later with biopsy-proven disease in the pelvis and lungs. She was treated with chemotherapy but died of disease 6 years later. The other recurrence was in a woman who had a conization with $<1 \mathrm{~mm}$ of squamous cell carcinoma. She subsequently underwent a laparoscopic simple hysterectomy, which showed $6 \mathrm{~mm}$ of invasion and negative margins. She was enrolled in the trial and underwent laparoscopic lymphadenectomy with 11 negative lymph nodes. She was diagnosed with a biopsy-proven inguinal lymph node recurrence 10 months later. She was treated with chemoradiation and is without evidence of disease after 4 years of follow-up. Following these two additional recurrences, the study was closed in 2016 according to the above noted stopping rule. The data were reviewed by the Data and Safety Monitoring Committee and the study was deemed safe to reopen provided that women who had an inadvertent simple hysterectomy were excluded due to their high recurrence rate $(2 / 16,12.5 \%)$. Of note, none of the recurrences occurred in the parametria.

Significant adverse events (CTCAE version 4.0 grade 4 to 5 ) were noted in two patients (2.0\%). One patient died 26 days after surgery (laparoscopic lymph node dissection) of a presumed postoperative venous thromboembolism. A second patient had significant bleeding 12 days post-operatively from conization, which required transfusion and reoperation with sutures placed in the cervix to control the bleeding.
To date, 14 pregnancies have been reported among 11 of 40 women $(27.5 \%)$ who underwent cervical conization and lymph node assessment for fertility preservation and remain in the study. Of these 14 pregnancies, $13(92.9 \%)$ delivered at term and one (7.1\%) resulted in a fetal demise at 22 weeks of gestation. It is unknown how many additional women attempted to become pregnant.

\section{DISCUSSION}

\section{Summary of Main Results}

The ConCerv Trial showed that conservative surgery with conization and simple hysterectomy is feasible in patients with early-stage, low-risk cervical carcinoma. The rate of positive lymph nodes was $5 \%$ and the rate of residual disease in the hysterectomy specimen following conization was $2.5 \%$. The 2-year recurrence rate was $3.5 \%$ overall; $2.4 \%$ (1/42) among patients who had conization; $0 \%$ $(0 / 36)$ among patients who had conization followed by hysterectomy; and $12.5 \%(2 / 16)$ among women who had an inadvertent simple hysterectomy. These results are similar to the findings from previous retrospective and small prospective studies described below. ${ }^{17} 19-222526$

\section{Results in Context of Published Literature}

Early studies by Rob et al ${ }^{19} 20$ reported the feasibility and safety of performing less radical, fertility-sparing surgery in women with FIG0 2009 stage IA1-IB1 cervical carcinoma. All patients underwent laparoscopic sentinel lymph node identification with frozen section. Of the 40 patients enrolled, 6 (15\%) had positive sentinel lymph nodes on frozen section, and radical hysterectomy with pelvic lymphadenectomy was immediately performed according to the local standard of care. In the remaining patients, only a pelvic lymphadenectomy was performed. Following a 7-day interval to allow pathologic confirmation of negative lymph nodes, a large cone or simple vaginal trachelectomy was performed. With a mean follow-up of 47 months, one recurrence was reported in a patient with a stage IB1 tumor with $8 \mathrm{~mm}$ of cervical stromal invasion and lymphovascular space invasion present. Of the 24 women who tried to conceive, 17 (71\%) became pregnant with 11 births. The authors concluded that large cone or simple trachelectomy with laparoscopic pelvic lymph node dissection was safe and feasible with a high pregnancy rate in women with early-stage cervical cancer. ${ }^{19} 20$ The same group evaluated less radical surgery in 60 women not desiring fertility preservation. ${ }^{21}$ All participants had FIG0 2009 stage IA1-IB1 cervical cancer with favorable pathologic characteristics (tumor size $<2 \mathrm{~cm}$ and $<50 \%$ stromal invasion) and underwent laparoscopic sentinel lymph node identification with frozen section. Five patients (8.3\%) had positive sentinel lymph nodes on frozen section and underwent radical hysterectomy with pelvic lymphadenectomy according to the local standard of care. In the remaining 55 patients, a complete pelvic lymphadenectomy and simple vaginal hysterectomy was performed. With a median follow-up of 47 months, no recurrences were reported. The authors concluded that simple hysterectomy with pelvic lymph node dissection was safe and feasible in select women with earlystage cervical cancer who did not desire fertility preservation. ${ }^{21}$ The ConCerv Trial showed similar findings, with no recurrences noted 


\section{Original research}

in the 40 women who underwent conization followed by simple hysterectomy and negative lymph node assessment.

A subsequent study by Plante et $\mathrm{al}^{17}$ evaluated 50 patients with early-stage low-risk cervical cancer who underwent a simple vaginal trachelectomy/conization with laparoscopic lymph node evaluation. Lymph nodes were negative in 46 patients $(92 \%)$, three patients had isolated tumor cells, and one patient had micrometastasis. Thirty patients $(60 \%)$ had either no residual disease or cervical dysplasia only in the simple trachelectomy specimen. With a median follow-up of 76 months, only one local recurrence was seen, which was treated initially with chemoradiation. This patient again had a local recurrence and underwent a pelvic exenteration, but the disease progressed and she died of disease. Forty pregnancies were reported and $75 \%$ delivered at term. ${ }^{17}$ Several recent retrospective analyses have also shown the safety and efficacy of conservative surgery. ${ }^{22} 2526$

Two large database studies and a systematic review evaluating conservative surgery in early-stage, low-risk cervical cancer have recently been published. ${ }^{27-29}$ Tseng et al ${ }^{27}$ used the Surveillance, Epidemiology, and End Results (SEER) database to evaluate 2717 patients with FIGO 2009 stage IB1 disease, all of whom had pelvic lymphadenectomy performed. They compared women who underwent uterine preserving surgery $(n=125)$ with conization or simple trachelectomy with women who underwent hysterectomy of any type $(n=2592)$. They noted no differences in 10-year disease-specific survival between the two groups. ${ }^{27} \mathrm{~A}$ subsequent study by Sia and colleagues ${ }^{28}$ used the National Cancer Database to compare outcomes between simple and radical hysterectomy for 1530 women with stage IA2 and 3931 women with stage IB1 disease. They noted no association between surgical radicality and survival for women with stage IA2 tumors. However, there was a $55 \%$ increase in mortality for women with stage IB1 disease who underwent simple compared with radical hysterectomy. ${ }^{28} \mathrm{~A}$ lymph node evaluation was not performed in $19 \%$ of patients with stage IB1 disease who underwent simple hysterectomy versus $2 \%$ of women with stage IB1 disease who underwent radical hysterectomy, raising the possibility of undiagnosed lymph node metastases and undertreatment in the adjuvant setting. After adjusting for nodal assessment, the difference in survival was no longer statistically significant. It is unknown how many women in this study had an inadvertent simple hysterectomy with an unexpected post-operative diagnosis of invasive cancer, potentially affecting the recurrence and survival rates as seen in the ConCerv Trial. ${ }^{28}$

A recently reported systematic review by Wu et a ${ }^{29}$ examined the outcomes of simple hysterectomy for low-risk, early-stage cervical cancer from 21 studies with a total of 2662 women. Most women $(96.8 \%)$ had tumors $<2 \mathrm{~cm}$, and $15.4 \%$ had tumors with lymphovascular space invasion. FIG0 stage was IA1 in $36.1 \%$ and IB1 in $61.0 \%$ of patients. The recurrence rate for the 19 studies reporting recurrence data was $5.4 \%$. The total death rate for the 20 studies reporting survival data was $5.5 \%$, encompassing $2.7 \%$ of patients with stage IA2 disease and $7.3 \%$ with stage IB1 disease. However, only $71.8 \%$ of patients had a lymph node assessment, with $3.2 \%$ exhibiting positive lymph nodes, limiting the conclusions that could be drawn from the study. ${ }^{29}$

In the ConCerv Trial, three patients developed recurrent disease, two patients with stage IB1 disease and one patient with stage IA2 disease. However, one patient had invasion $>10.0 \mathrm{~mm}$ as well as positive cone margins for high-grade dysplasia and the other two patients had an inadvertent simple hysterectomy. The inclusion criteria for the trial were changed based on these findings as described above. Of note, none of the recurrences occurred in the parametria. Five per cent of patients had positive lymph nodes. This is similar to the findings of Park et al, ${ }^{30}$ suggesting that lymph node assessment with sentinel lymph node biopsy and/or full lymph node dissection should be performed in this population. This is in accordance with current guidelines from the National Comprehensive Cancer Network and the European Society for Medical Oncology, which recommend lymph node assessment in all patients undergoing hysterectomy for cervical cancer. ${ }^{31} 32$

The publication of the Laparoscopic Approach to Cervical Cancer (LACC) Trial ${ }^{33}$ occurred during the last year of enrollment of the ConCerv Trial. The LACC Trial was a prospective, randomized study which showed that minimally invasive radical hysterectomy is associated with lower rates of disease-free survival and overall survival compared with open abdominal radical hysterectomy among women with early-stage cervical cancer. The results of the LACC Trial have changed the standard of care for women with earlystage cervical cancer, with minimally invasive surgery no longer recommended in women undergoing radical hysterectomy. ${ }^{33}$ These results did not significantly impact the ConCerv Trial or require a change in our protocol as almost all study procedures were already completed at the time of the LACC Trial publication. However, it is important to note that $96 \%$ of the patients on the ConCerv Trial underwent minimally invasive surgery. Of the 56 patients who underwent a simple hysterectomy, the majority $(40 / 56,71.4 \%)$ had a cone with removal of all tumor prior to undergoing hysterectomy and lymph node assessment. As shown in Figure 1, none of these patients developed a recurrence within the 2-year follow-up period. However, of the 16 patients who had undergone an inadvertent simple hysterectomy prior to study enrollment, two (12.5\%) developed recurrent disease. Both patients underwent minimally invasive surgery for both the simple hysterectomy and the pelvic lymph node dissection. The role of minimally invasive surgery for conservative surgery, including both simple hysterectomy after a conization with negative margins as well as for lymph node assessment (sentinel lymph node biopsy and/or full pelvic lymph node dissection), remains unclear and requires further study.

\section{Strengths and Weaknesses}

The strengths of our study include that it is the first comprehensive prospective evaluation of conservative surgery in patients with lowrisk cervical cancer. Furthermore, all surgical specimens underwent central pathology review by an expert gynecologic pathologist. In addition, all study data were entered into a central REDCap database and the quality and safety of the study procedures were closely monitored by the MD Anderson coordinating center as well as the Data and Safety Monitoring Committee. An additional strength is that the trial included multiple sites from low-resource regions, which have a high prevalence of cervical cancer. This allowed us to show that conservative surgery is safe and feasible in both highand low-resource settings. Furthermore, the study allowed us to build a robust network of collaborators around the globe, facilitating a pathway for future treatment trials with participants from regions with a high burden of disease. 
Our study is limited by a prolonged study period of almost 9 years. This was primarily due to the strict inclusion criteria, requirement for central pathology review, and limited number of women meeting the strict eligibility criteria. To overcome these barriers, the study was opened in several sites with the associated challenges of working across multiple countries with different time zones, languages, and regulations related to securing contracts and obtaining institutional review board approvals. Every amendment to the protocol or informed consent required translation and approval from each participating site, often resulting in delays and pauses in the study. During this long study period, there were changes in the standard of care for the management of cervical cancer, including the introduction of sentinel lymph node biopsy. ${ }^{34}$ This change in practice was implemented at some, but not all, participating sites, and at different time points based on local guidelines, availability of specialized equipment/dyes and surgeon training. As a result, the lymph node assessment (sentinel lymph node biopsy and/or full pelvic lymph node dissection) was not consistent across sites. Similarly, the choice of surgical approach (open, laparoscopic, or robotic) for both the simple hysterectomy and lymph node assessment was based on surgeon preference and training, and also not consistent across sites. Our study protocol required at least two separate surgeries with one (and sometimes two) conization procedures to confirm eligibility for conservative surgery, followed by definitive therapy with simple hysterectomy and/or lymph node assessment. Our group previously reported a single-step procedure with conization and frozen section for intra-operative triage of simple versus radical hysterectomy in 150 women with stage IA1 disease ${ }^{35}$ However, further study is needed to determine the safety of this single-step approach for patients potentially eligible for conservative surgery, particularly in institutions without specialized pathology services as well as in low- and middle-income countries.

Another important limitation of the ConCerv Trial is that the inclusion criteria were amended during the course of the trial. As described above, this was prompted by three patients developing recurrent disease. The first recurrence occurred very early in the study (2010) and was felt to be a study design flaw, with inadequate inclusion criteria. The requirements for depth of invasion $<10 \mathrm{~mm}$ and negative cone margins for high-grade dysplasia were added. In 2016, the study was stopped because two additional patients developed recurrent disease, both of whom had undergone an inadvertent simple hysterectomy. After extensive review, the Data and Safety Monitoring Committee approved reopening the study provided that we excluded women who had undergone a simple hysterectomy without a prior cone with negative margins. There is no current standard of care for this group of patients who undergo inadvertent simple hysterectomy, and the role of conservative surgery with lymphadenectomy remains unclear in this patient population.

\section{Implications for Practice and Future Research}

In addition to the ConCerv Trial, two ongoing prospective studies are evaluating conservative surgery in low-risk cervical cancer. The Radical versus Simple Hysterectomy and Pelvic Node Dissection with Low-Risk Early-Stage Cervical Cancer (SHAPE) Trial (NCT01658930) is a non-inferiority randomized phase III study comparing simple hysterectomy plus pelvic lymph node dissection with radical hysterectomy plus pelvic lymph node dissection in patients with FIGO 2009 stage IA2-IB1 disease (tumors $<2 \mathrm{~cm}$ ). The primary outcomes are safety and pelvic relapse-free survival. The Gynecologic Oncology Group (GOG) 278 Trial (NCT01649089) is assessing the impact of non-radical surgery (simple hysterectomy or cone biopsy, both with lymphadenectomy) on functional outcomes of lymphedema, bladder, bowel, and sexual function in women with FIG0 2009 stage IA2-IB1 (tumors $\leq 2 \mathrm{~cm}$ ) cervical cancer. Secondary outcomes include recurrence and survival rates. Both of these studies are nearing completion and will be reported in the near future.

The results of the ConCerv Trial have shown that conservative surgery in patients with low-risk cervical cancer may be a feasible and oncologically safe option. This includes conization alone or conization followed by simple hysterectomy, both with lymph node assessment. As such, these results should be considered and discussed with patients who meet low-risk criteria as outlined in our study. If the SHAPE and GOG 278 studies show similar results, the standard of care may change from radical hysterectomy to conservative surgery with conization in women desiring fertility preservation, and simple hysterectomy in women who have completed childbearing. In all cases, pelvic lymph node assessment with sentinel lymph node biopsy and/or full pelvic lymph node dissection is still recommended based on the results of the ConCerv Trial and others.

\section{CONCLUSIONS}

In summary, the results of the ConCerv Trial suggest that conservative surgery for women with early-stage, low-risk cervical cancer is safe and feasible. Further investigation is still needed to address several unanswered questions including the long-term outcomes of conservative surgery; the role of a minimally invasive approach in conservative surgery; the impact on quality of life; and the best management for women who undergo an inadvertent simple hysterectomy with a post-operative diagnosis of cervical cancer. Furthermore, we need to continue to study and refine the optimal pathologic criteria for conservative surgery. Findings from the ConCerv Trial offer prospective data supporting a more conservative approach to low-risk patients, sparing them the early and late morbidity associated with radical procedures. It will also allow for safer cervical cancer surgery in low- and middle-income countries, where the burden of cervical cancer is highest.

\footnotetext{
Author affiliations

${ }^{1}$ Department of Gynecologic Oncology and Reproductive Medicine, The University of Texas MD Anderson Cancer Center, Houston, Texas, USA

${ }^{2}$ Instituto Nacional de Cancerología, Bogotá, and Clínica de Oncología Astorga, Medellin, Colombia

${ }^{3}$ Instituto Nacional de Enfermedades Neoplásicas, Lima, Peru

${ }^{4}$ A.C. Camargo Cancer Center, A.C. Camargo Cancer Center, Sao Paulo, Brazil

${ }^{5}$ Instituto Brasileiro de Controle do Cancer, Sao Paulo, Brazil

${ }^{6}$ Hospital Italiano de Buenos Aires, Buenos Aires, Argentina

${ }^{7}$ Hospital Erasto Gaertner, Curitiba, Brazil

${ }^{8}$ Instituto Nacional de Cancerologia, Mexico, Mexico

${ }^{9}$ Chulalongkorn University and King Chulalongkorn Memorial Hospital, Bangkok, Thailand

${ }^{10}$ Nebraska Methodist Health System, Omaha, Nebraska, USA

${ }^{11}$ Royal Women's Hospital and University of Melbourne, Melbourne, Victoria, Australia

${ }^{12}$ Instituto de Ginecología de Rosario, Rosario, Argentina

${ }^{13}$ Fondazione Policlinico Universitario A. Gemelli IRCCS and Catholic University of the Sacred Heart, Rome, Italy
} 


\section{Original research}

\author{
${ }^{14}$ Matamoros General Hospital Alfredo Pumarejo, Matamoros, Mexico \\ ${ }^{15}$ Hospital Británico de Buenos Aires, Buenos Aires, Argentina \\ ${ }^{16}$ Instituto de Cancerologia - Las Américas - AUNA, Medellin, Colombia \\ ${ }^{17}$ Department of Pathology, University of Texas MD Anderson Cancer Center, \\ Houston, Texas, USA \\ ${ }^{18}$ Department of Biostatistics, University of Texas MD Anderson Cancer Center, \\ Houston, Texas, USA \\ ${ }^{19}$ US Oncology Research, The Woodlands, Texas, USA
}

\section{Presented at}

This study was presented in part at the annual meeting of the International Gynecologic Cancer Society (IGCS), Rio de Janeiro, Brazil, September 2019.

\section{Twitter Andre Lopes @Andrelopes1002, Michael Frumovitz @frumovitz and Pedro} T Ramirez @pedroramirezMD

Acknowledgements The authors would like to thank the following research personnel and collaborators for their assistance in recruiting patients, collecting/ entering data, and working across different countries, languages, and time zones to complete this study: MD Anderson Cancer Center, Houston, Texas, USA: Cindy Melendez, Jessica Gallegos, Juana Rayo, Ana Lopez, Keiry Paiz, and Mark Munsell; Instituto de de Cancerología - Las Américas - AUNA, Medellín, Colombia: Natalia Escobar; Instituto Nacional de Enfermedades Neoplásicas (INEN), Lima, Perú: Ninoska Macavilca; Barretos Cancer Hospital, Barretos, Brazil: Talita Garcia, Julio Cesar Souza, Ligia Zampieri, Carlos Andrade, Georgia Fontes-Cintra, Marcelo Viera, Ricardo dos Reis, Hospital Italiano de Buenos Aires, Buenos Aires, Argentina: Mariana Prada, Marina Lamm, and Ana Jaen; Instituto Nacional de Cancerologia, México City, México: Lenny Gallardo, Instituto Brasileiro de Controle do Cancer (IBCC), São Paulo, Brazil: Alayne Domingues Yamada; Hospital Erasto Gaertner, Curitiba, Brazil: Nathalia Carneiro, Fernanda Schamne; Chulalongkorn University and King Chulalongkorn Memorial Hospital, Bangkok, Thailand: Patou Tantbirojn, MD; Nebraska Methodist Health System, Omaha, Nebraska, USA: Kathryn Bartz, RN; Royal Women's Hospital, Melbourne, Australia: Julie Silvers; Fondazione Policlinico Universitario A. Gemelli IRCCS and Catholic University of the Sacred Heart, Rome, Italy: Luigi Carlo Turco, Anna Fagotti, Francesco Cosentino, Instituto de Ginecología de Rosario, Rosario, Argentina: Gabriela Santillan, and Jeronimo Costa. The authors would also like to thank all the women who entrusted us with their care and participated in the study in order to improve the care of women with cervical cancer globally.

Contributors Conception and design: KMS, PTR, MF, RP, RLC. Administrative support: KMS, PTR, RLC. Provision of study material or patients: all authors. Collection and assembly of data: KMS, BMF, PR, PTR, RP, RLC. Data analysis and interpretation: BMF, KMS, PTR, MF, RP, RLC. Manuscript writing: all authors. Final approval of manuscript: all authors. Accountable for all aspects of the work: all authors.

Funding This research was supported in part by the National Institutes of Health (NIH) through MD Anderson's Cancer Center Support Grant P30CA016672 and the MD Anderson Sister Institution Network Fund.

Competing interests The authors have the following disclosures: $\mathrm{MH}$ : consulting/ speaker for Stryker and research support from GlaxoSmithKline and Astra Zeneca.

Patient consent for publication Not required.

Provenance and peer review Not commissioned; externally peer reviewed.

Data availability statement Data are available upon reasonable request. In accordance with the journal's guidelines, we will provide our data for the reproducibility of this study in other centers if such is requested. The data are in a REDCap database at MD Anderson Cancer Center and are available from the principal investigator, Dr. Kathleen Schmeler.

\section{ORCID iDs}

Kathleen M Schmeler http://orcid.org/0000-0002-9670-4189

Rene Pareja http://orcid.org/0000-0003-0093-0438

Gabriel J Rendon http://orcid.org/0000-0002-7536-0567

Michael Frumovitz http://orcid.org/0000-0002-0810-2648

\section{REFERENCES}

1 Arbyn M, Weiderpass E, Bruni L, et al. Estimates of incidence and mortality of cervical cancer in 2018: a worldwide analysis. Lancet Glob Health 2020;8:e191-203.
2 Bray F, Ferlay J, Soerjomataram I, et al. Global cancer statistics 2018: GLOBOCAN estimates of incidence and mortality worldwide for 36 cancers in 185 countries. CA Cancer J Clin 2018:68:394-424.

3 Organization TWH. Global strategy to accelerate the elimination of cervical cancer as a public health problem, 2018. Available: https:// www.who.int/publications/i/item/9789240014107

4 Piver MS, Rutledge F, Smith JP. Five classes of extended hysterectomy for women with cervical cancer. Obstet Gynecol 1974;44:265-72.

5 Plante M, Gregoire J, Renaud M-C, et al. The vaginal radical trachelectomy: an update of a series of 125 cases and 106 pregnancies. Gynecol Oncol 2011;121:290-7.

6 Low JA, Mauger GM, Carmichael JA. The effect of Wertheim hysterectomy upon bladder and urethral function. Am J Obstet Gynecol 1981;139:826-30.

7 Kadar N, Saliba N, Nelson JH. The frequency, causes and prevention of severe urinary dysfunction after radical hysterectomy. $\mathrm{Br} \mathrm{J}$ Obstet Gynaecol 1983;90:858-63.

8 Sood AK, Nygaard I, Shahin MS, et al. Anorectal dysfunction after surgical treatment for cervical cancer. J Am Coll Surg 2002;195:513-9.

9 Bergmark K, Avall-Lundqvist E, Dickman PW, et al. Vaginal changes and sexuality in women with a history of cervical cancer. $N$ Engl J Med 1999;340:1383-9.

10 Frumovitz M, Sun CC, Schover LR, et al. Quality of life and sexual functioning in cervical cancer survivors. J Clin Oncol 2005;23:7428-36.

11 Kinney WK, Hodge DO, Egorshin EV, et al. Identification of a low-risk subset of patients with stage $\mathrm{lb}$ invasive squamous cancer of the cervix possibly suited to less radical surgical treatment. Gynecol Oncol 1995;57:3-6.

12 Covens A, Rosen B, Murphy J, et al. How important is removal of the parametrium at surgery for carcinoma of the cervix? Gynecol Oncol 2002;84:145-9.

13 Stegeman M, Louwen M, van der Velden J, et al. The incidence of parametrial tumor involvement in select patients with early cervix cancer is too low to justify parametrectomy. Gynecol Oncol 2007;105:475-80.

14 Wright JD, Grigsby PW, Brooks R, et al. Utility of parametrectomy for early stage cervical cancer treated with radical hysterectomy. Cancer 2007;110:1281-6.

15 Frumovitz M, Sun CC, Schmeler KM, et al. Parametrial involvement in radical hysterectomy specimens for women with early-stage cervical cancer. Obstet Gynecol 2009;114:93-9.

16 Smith AL, Frumovitz M, Schmeler KM, et al. Conservative surgery in early-stage cervical cancer: what percentage of patients may be eligible for conization and lymphadenectomy? Gynecol Oncol 2010;119:183-6.

17 Plante M, Renaud M-C, Sebastianelli A, et al. Simple vaginal trachelectomy in women with early-stage low-risk cervical cancer who wish to preserve fertility: the new standard of care? Int $J$ Gynecol Cancer 2020;30:981-6.

18 Ramirez PT, Pareja R, Rendón GJ, et al. Management of low-risk early-stage cervical cancer: should conization, simple trachelectomy, or simple hysterectomy replace radical surgery as the new standard of care? Gynecol Oncol 2014;132:254-9.

19 Rob L, Charvat M, Robova $\mathrm{H}$, et al. Less radical fertility-sparing surgery than radical trachelectomy in early cervical cancer. Int $J$ Gynecol Cancer 2007;17:304-10.

20 Rob L, Pluta M, Strnad P, et al. A less radical treatment option to the fertility-sparing radical trachelectomy in patients with stage I cervical cancer. Gynecol Oncol 2008;111:S116-20.

21 Pluta M, Rob L, Charvat M, et al. Less radical surgery than radical hysterectomy in early stage cervical cancer: a pilot study. Gynecol Oncol 2009;113:181-4.

22 Martinelli F, Ditto A, Filippi F, et al. Conization and lymph node evaluation as a fertility-sparing treatment for early stage cervical cancer. Int J Gynecol Cancer 2021;31:457-61.

23 Thall PF, Wathen JK, Bekele BN, et al. Hierarchical Bayesian approaches to phase II trials in diseases with multiple subtypes. Stat Med 2003;22:763-80.

24 Harris PA, Taylor R, Thielke R, et al. Research electronic data capture (REDCap)--a metadata-driven methodology and workflow process for providing translational research informatics support. J Biomed Inform 2009;42:377-81.

25 Nica A, Marchocki Z, Gien LT, et al. Cervical conization and lymph node assessment for early stage low-risk cervical cancer. Int $J$ Gynecol Cancer 2021;31:447-51.

26 Fanfani F, Pedone Anchora L, Di Martino G, et al. Oncologic and obstetric outcomes after simple conization for fertility-sparing 
surgery in FIGO 2018 stage IB1 cervical cancer. Int J Gynecol Cancer 2021;31:452-6.

27 Tseng JH, Aloisi A, Sonoda Y, et al. Less versus more radical surgery in stage IB1 cervical cancer: a population-based study of long-term survival. Gynecol Oncol 2018;150:44-9.

28 Sia TY, Chen L, Melamed A, et al. Trends in use and effect on survival of simple hysterectomy for early-stage cervical cancer. Obstet Gynecol 2019;134:1132-43.

29 Wu J, Logue T, Kaplan SJ, et al. Less radical surgery for earlystage cervical cancer: a systematic review. Am J Obstet Gynecol 2021;224:348-58.

30 Park J-Y, Kim D-Y, Kim J-H, et al. Outcomes after radical hysterectomy according to tumor size divided by $2-\mathrm{cm}$ interval in patients with early cervical cancer. Ann Oncol 2011;22:59-67.
31 Koh W-J, Abu-Rustum NR, Bean S, et al. Cervical cancer, version 3.2019, NCCN clinical practice guidelines in oncology. J Natl Compr Canc Netw 2019;17:64-84.

32 Marth C, Landoni F, Mahner S, et al. Cervical cancer: ESMO clinical practice guidelines for diagnosis, treatment and follow-up. Ann Oncol 2017;28:iv72-83.

33 Ramirez PT, Frumovitz M, Pareja R, et al. Minimally invasive versus abdominal radical hysterectomy for cervical cancer. N Engl J Med 2018;379:1895-904.

34 Salvo G, Ramirez PT, Levenback CF, et al. Sensitivity and negative predictive value for sentinel lymph node biopsy in women with earlystage cervical cancer. Gynecol Oncol 2017;145:96-101.

35 Martinelli F, Schmeler KM, Johnson C, et al. Utility of conization with frozen section for intraoperative triage prior to definitive hysterectomy. Gynecol Oncol 2012;127:307-11. 